\title{
Moral experience: a framework for bioethics research
}

\author{
Matthew R Hunt, ${ }^{1,2}$ Franco A Carnevale ${ }^{3,4}$
}

${ }^{1}$ Centre de recherche en éthique (CRÉUM), University of Montreal, Montreal, Quebec Canada

${ }^{2}$ Department of Clinical Epidemiology and Biostatistics, McMaster University, Hamilton, Ontario, Canada

${ }^{3}$ School of Nursing, McGill University, Montreal, Quebec, Canada

${ }^{4}$ Pediatric Ethics Committee, McGill University Health Centre, Montreal, Quebec, Canada

\section{Correspondence to}

Dr Matthew R Hunt, Centre de recherche en éthique (CRÉUM), University of Montreal, C.P.

6128, succ. Centre-ville, Montreal, OC H3C 3J7, Canada; 1matthew.hunt@gmail.com

Received 14 July 2010 Revised 2 February 2011 Accepted 2 March 2011 Published Online First 22 April 2011

\section{ABSTRACT}

Theoretical and empirical research in bioethics frequently focuses on ethical dilemmas or problems. This paper draws on anthropological and phenomenological sources to develop an alternative framework for bioethical enquiry that allows examination of a broader range of how the moral is experienced in the everyday lives of individuals and groups. Our account of moral experience is subjective and hermeneutic. We define moral experience as "Encompassing a person's sense that values that he or she deem important are being realised or thwarted in everyday life. This includes a person's interpretations of a lived encounter, or a set of lived encounters, that fall on spectrums of right-wrong, good-bad or just-unjust". In our conceptualisation, moral experience is not limited to situations that are heavily freighted with ethically-troubling ramifications or are sources of debate and disagreement. Important aspects of moral experience are played out in mundane and everyday settings. Moral experience provides a research framework, the scope of which extends beyond the evaluation of ethical dilemmas, processes of moral justification and decision-making, and moral distress. This broad research focus is consistent with views expressed by commentators within and beyond bioethics who have called for deeper and more sustained attention in bioethics scholarship to a wider set of concerns, experiences and issues that better captures what is ethically at stake for individuals and communities. In this paper we present our conceptualisation of moral experience, articulate its epistemological and ontological foundations and discuss opportunities for empirical bioethics research using this framework.

\section{INTRODUCTION}

Everyday life is infused with moral implications and moral content. It is not only situations when the moral stakes are high for those involved or at critical decision points that are deserving of attention and ethical analysis. However, this is sometimes how ethics are discussed, particularly in the context of health and healthcare. In 1971 Edmund Pincoffs offered a critique of the tendency for scholarly discourse and analysis in the field of ethics to adopt a narrow focus on moral justification, ethical problems and rationales for decisionmaking. ${ }^{1}$ Pincoffs identified a preoccupation with 'quandary ethics' and suggested that broader engagement was needed with other aspects of ethics and the moral life. The prevailing discourse within the bioethics literature has continued to focus on ethical dilemmas and problems, as well as moral distress. In addition, much of the content of ethics education for health professionals is focused on analysing and resolving dilemmas. Clearly, these are deeply important topics and bioethics scholars should engage in this analysis. However, this dominant focus may also allow less room for considering other dimensions of how individuals and communities understand and experience the moral in their everyday lives. Indeed, the scope of bioethics and the ambit of bioethics scholarship encompasses more than the processes of evaluating options and enacting choices in response to one's understanding of a contentious or vexing situation. This reality has implications for both empirical and theoretical approaches to bioethics. Drawing attention to a broader array of moral experience can lead to better understanding and richer analysis of topics of concern in bioethics.

In addition to the primacy of 'quandary ethics', other lines of critique have been expressed by commentators within and outside the field of bioethics. Scholars have encouraged greater recognition and accounting for context and social relationships in ethical enquiry, ${ }^{23}$ and conceptualisation of persons as being more than isolated, rational thinkers. ${ }^{4}$ The value and the possibility of such enquiry has been demonstrated by bioethics researchers who have conducted theoretical and empirical analyses that illuminate ethical dimensions of heath-related experiences that go beyond dilemmas and the dominant issues of medical ethics (eg, confidentiality, informed consent). ${ }^{56}$

Critics from beyond bioethics have also argued that the traditional preoccupation of bioethics with the tools and approaches of philosophy and the lesser role played by the contextualising disciplines of anthropology and sociology limited the ability of bioethics scholarship to provide a more full and robust accounting for the moral dimensions of everyday life. $^{7}{ }^{8}$ The increasing participation of social scientists in bioethics has contributed to a broadened scope of enquiry, and played a role in moving the field even further beyond a narrow focus on quandary ethics. ${ }^{9}$ Over the past decade empirical approaches to bioethics enquiry have occupied an increasingly prominent place in bioethics scholarship. ${ }^{10}{ }^{11}$ Empirical bioethics aims to "produce a contextualised ethical analysis, which is both sensitive to the lived experiences of stakeholders and yet still critically normative'. ${ }^{11}$ (p.1)

In this article we present a conception of 'moral experience' for bioethics research. Our aim is to articulate a theoretical frame for bioethics scholars seeking to access and better understand how individuals live out the moral dimensions of their lives. In doing so, we draw on a range of anthropological and phenomenological sources. The term 'moral experience' has a multiplicity of meanings; at present there is no shared definition of this term. Marcel Mandelbaum, in his book The Phenomenology of Moral Experience, focuses on moral judgements and obligations. ${ }^{12}$ Arthur Kleinman and colleagues 
have articulated a concept of moral experience that more closely approximates our analysis. In an article examining moral experience, culture and stigma, they define moral experience as '... what is most at stake for actors in a local social world'. ${ }^{13}$ (p. 1525) Elsewhere Kleinman states that '...moral experience is about the local processes (collective, interpersonal, subjective) that realize (enact) values in ordinary living'. (p. 71) In using moral experience in these ways, these scholars articulate an understanding of the moral life of individuals as being shaped and influenced by the moral experience of local networks, neighbourhoods and communities. Of key importance is the assertion that the moral experience of individuals-what matters most to them-cannot be understood without considering the local social worlds that they inhabit. Moral experience is centred on what matters most to individuals and is shaped by the interaction of three phenomena: 'cultural meanings, social experience and subjectivity (inner emotions and sense of self)'. ${ }^{14}$ We agree with the necessity of examining the situated, embedded nature of individuals within groups and communities, and the diverse sources that influence individual moral experience. In this article we present an account of moral experience that draws inspiration from Kleinman's framework and is hermeneutical. We develop a conception of moral experience for the purpose of offering conceptual resources for empirical and theoretical enquiry in bioethics; our use of moral experience aims to provide a vocabulary and framework for considering a wider compass of understandings and experiences in the moral lives of individuals and groups.

\section{PHILOSOPHICAL FRAMEWORK}

To develop our conceptualisation of moral experience, we draw on the conception of the human sciences articulated by Taylor. ${ }^{15}$ Building on the work of Heidegger ${ }^{16}$ and Gadamer, ${ }^{17}$ Taylor has argued for an ontological shift in our understanding of human phenomena. In contrast to physical, chemical or biological phenomena that arguably exist in a relatively fixed manner regardless of how we conceive them, understanding human agency involves the discernment of phenomena in terms of their broader meaningfulness. ${ }^{15}$ This process involves seeking to understand how they matter to the agent, which is in part shaped by the systems of meaning the agent is socially embedded within. Thus we regard moral experience as a contextualised experiential phenomenon that is best understood from the subjective perspective of the person living the phenomenon within his or her local social context. In contrast, a positivist framework would construe a phenomenon in mechanistic objective terms.

Within this contextualised subjectivist consideration of the human sciences, Taylor has elaborated a moral framework which is particularly helpful in developing the conception of moral experience we are advancing here. Moral matters are rooted in a person's moral ontology-that is, the underlying commonly implicit beliefs held by a person or a group of persons. In this way the person as a moral agent stands against a background 'horizon of significance' constituted by the context that the agent resides within and the sociohistorically-based moral order within which meaning is rooted for her or him. ${ }^{18}$ Subjective experience is rooted in horizons of significance that shape how things matter to individual persons and individual experience is enacted within a particular context of meanings-what Geertz has described as 'webs of significance'. ${ }^{19}$ Historical, cultural and social processes continually affect the value assigned to various aspects of everyday life. Highly-held 'goods' within social communities-expressed in terms of beliefs and values-are derived from their shared background horizon of significance. Each person is 'thrown' within particular horizons of meanings against which he or she continually assesses how things matter. $^{16}$

Although moral experience involves a sense of how things matter in relation to a person's value reference systems, this framework strives to understand the perspective of individual experience within that local social world. How a person thinks and feels about particular phenomena are shaped in relation to surrounding conceptions of the good. Taylor argues that the human agent enacts 'personal' choices and actions in light of the meaningful context within which the agent resides. ${ }^{18}$ The surrounding community or society elaborates its own relative order of various 'goods' by discerning which actions or modes of life are morally higher through processes of 'distinctions of worth' ${ }^{20}$ Agents cannot stand outside their respective horizon of significance and their corresponding moral framework. ${ }^{21}$

Human experience entails a continual appraisal of how individual concerns stand in relation to the local moral context. The conception of moral experience we develop below is rooted in this philosophical framework. Moreover, understanding phenomena within this framework entails hermeneutical interpretation. ${ }^{16172022}$ Hermeneutics refers to the 'science or art' of interpretation. ${ }^{1723}$ Hermeneutic 'interpretation is an attempt to make clear, to make sense of an object of study... The interpretation aims to bring to light an underlying coherence or sense'. ${ }^{22}$ (p. 16) Hermeneutical interpretation seeks clarity by identifying the object in which clarity is sought, distinguishing this underlying clarity from its presenting expression and specifying the subject for whom the underlying clarity is meaningful. Interpretation entails a 'hermeneutical circle', examining 'part-whole' relations, seeking the sense of the whole through an examination of its parts that is continually related back to the whole. For moral matters this involves seeking the underlying sense-that is, the agent's moral ontological horizon of significance corresponding with the moral matter in question. Horizons shape human agency. A person's identity and sense of worth is rooted in the background horizon of significance. ${ }^{18}$ Examining one's respective horizon of significance through hermeneutical interpretation helps to clarify where an agent stands in relation to the good.

The development of a conceptualisation of moral experience and the discussion of subjective experience presented here intersect with ideas in the philosophy of emotions. ${ }^{24-26}$ A detailed analysis of these intersections, however, is beyond the scope of this paper.

\section{ELABORATING A CONCEPTION OF MORAL EXPERIENCE}

We propose the following conceptualisation of moral experience:

Moral experience encompasses a person's sense that values that he or she deems important are being realised or thwarted in everyday life. This includes a person's interpretations of a lived encounter, or a set of lived encounters, that fall on spectrums of right-wrong, good-bad or just-unjust.

\section{Sense and interpretation}

Our conceptualisation draws upon Kleinman's use of 'moral experience', which highlights the subjective experience of individuals within local social worlds as well as a hermeneutic understanding of lived experience within particular horizons of significance, ${ }^{18}$ with the goal of articulating a framework for conducting bioethics enquiry. In line with this hermeneutical understanding, the conceptualisation that we develop specifies 
that moral experience encompasses the 'sense' and 'interpretation' of particular encounters by individuals, and the meanings they ascribe to particular experiences.

\section{Person}

We focus on a person's experiences and the interpretations he or she makes of what matters for him or her in particular circumstances. However, subjective experience cannot be evacuated from relationships, collective histories or context that constrain or shape it. Attention is given to the complex matrix of social, cultural and historical influences-that is, horizons of significance. Thus, while our conceptualisation of moral experience is hermeneutical and therefore focuses on individual persons, we do not intend to deny or disregard the critical role of collective experience.

In some settings we believe that it is possible to identify the moral experience of a group. An obvious example is the moral experience of a family; other examples include a treatment team or patients on a long-term care ward. A family's moral experience would be more than (and different from) the sum of the moral experiences of individual family members. The family group can be thought of as a social microsystem where a collective moral experience is shared and can be examined. Investigation into the moral experience of a family would examine the moral experience of individual members. Consideration would be given to how these experiences and interpretations are shared or diverge, and how family members contribute to the collective social experience, shared meanings and identity of being part of the family. ${ }^{14}$

\section{Values thwarted and realised in everyday life}

Moral experience encompasses an individual's sense that meaningful values are being enacted or impeded. It is not only when particular values are in jeopardy or tension that is of interest within a moral experience framework; situations when values are actualised are also considered to be aspects of moral experience worthy of attention. In this way, it is not just an experience of injustice that counts as moral experience; a sense of a situation being fair or equitable would also be understood as a moral experience. We also view a diffuse feeling of unfairness as a moral experience that needs to be accounted for, in addition to a sharply focused-and acutely felt-sense of manifest injustice. Even a sense of ambivalence or uncertainty about a lived encounter might be included here, particularly when the person identifies the experience as having significance in relation to values that matter for him or her.

Moral experience can help to understand the myriad ways that different experiences and normative evaluations may interrelate, since aspects of moral experience are rarely fully separate or dissociable. For example, a parent's guilt associated with their child's suffering is entwined with their conception of what it means to be a good parent. Our understanding is enriched when we can grasp these interrelations.

\section{Lived encounter or set of lived encounters}

Interpretations are made of lived encounters or a set of lived encounters. In our conceptualisation of moral experience, the sense that values are realised or thwarted may or may not be linked to an identifiable object. For example, a person may feel that a specific institutional arrangement is unjust. Another person may have a generalised feeling that life is unfair. Both of these feelings can appropriately be called a moral experience. In addition, overarching themes or orientations in a person's outlook also influence how an individual experiences particular phenomena or circumstances (eg, an individual's experiences may be influenced by a belief that 'every cloud has a silver lining' or 'suffering is redemptive'). Such background orientations are closely entwined with moral experience.

\section{Spectrums of right-wrong, good-bad, just-unjust}

The three spectrums of right-wrong, good-bad and just-unjust are intended to capture a wide array of experiences to which an individual ascribes moral significance. In describing spectrums rather than categories (good or bad, right or wrong, just or unjust), the variations and degrees of moral experiences are made visible. This approach also draws attention to aspects of moral experience that are played out in seemingly mundane and everyday settings. Other terms could also be associated with aspects of moral experience-or emotional responses to moral experience-such as distressed or fulfilled conscience, remorse, regret, obligation, satisfaction, guilt, ambivalence, compromise and responsibility. While these terms may alert us to the presence of moral experiences, they do not represent the core aspect of this concept.

\section{MORAL EXPERIENCE AS A FRAMEWORK FOR ENOUIRY IN EMPIRICAL BIOETHICS}

Moral experience can be used in empirical bioethics enquiry to provide an angle of vision to explore how individuals or groups live out the moral dimensions of a particular set of encounters. Given that this is a hermeneutical conception of moral experience, empirical research based on moral experience should be qualitative and interpretive. As such, not all methodological frameworks will be compatible with the conception of moral experience outlined here. For example, moral experience is inconsistent with a positivist research design. To achieve epistemological coherence within the project, and to match methodology and research question, the chosen approach should be oriented by a constructivist paradigm. Constructivism holds that knowledge is socially constructed, that researcher and researched-into co-create the study findings, and that a naturalistic and dialectic approach is necessary as no a priori theory will adequately capture the subjective phenomenon of interest. ${ }^{27}$ In addition, methodologies that are consistent with a hermeneutic approach will provide a close fit for enquiry into moral experience as we have conceptualised it here. A hermeneutic phenomenology such as interpretive phenomenology ${ }^{28}$ would be an obvious choice, but other options such as interpretive description ${ }^{29}$ and naturalistic enquiry ${ }^{30}$ could be considered. In addition, ethnographic approaches may also be consistent with an enquiry into moral experience as articulated here. Ultimately, the researcher should ensure that all aspects of the enquiryincluding research objective and questions, theoretical underpinnings, methodology and methods of data collection and analysis-are compatible with each other. ${ }^{31}$

We describe two qualitative research projects that we have conducted to illustrate how we have used moral experience to orientate empirical enquiry.

Carnevale and colleagues conducted a study of the moral experience of families with a child living at home whose breathing was ventilator-assisted. ${ }^{32}$ Interviews were conducted with the children and family members as well as clinicians who provided care for these children. Observations were also done in the home setting. Data analysis revealed a range of themes that included confronting parental responsibility, seeking normality, conflicting social values, living in isolation, the voice of children and questioning the moral order. The overarching phenomenon 
that was identified through this analysis was that these families experienced both distress and enrichment on a daily basis. It became apparent that the moral lives of these families cannot be apprehended without acknowledging both the distress and enrichment that are key and intertwined facets of their moral experience. Challenging common conceptions of living at home with assisted ventilation that construe such lives as extraordinarily burdensome for both patients and their families, the study demonstrated that the difficulties they confronted were intertwined with under-recognised sources of fulfilment such as the enjoyment of everyday pleasures for the patient, caring for one's child as a parent and reciprocal exchanges of affection among siblings. For example, a major challenge for parents was the possibility of not providing the ventilated child the care and attention that he or she required-striving to be a good parent. Our hermeneutical analysis of families' difficulties sought to better understand how these mattered to each family, revealing that these difficulties were embedded in families' fundamental values and commitments.

In a second study Hunt examined the moral experience of Canadian healthcare professionals who participated in humanitarian work. ${ }^{33}$ Data included in-depth interviews, blogs and published narrative accounts. Analysis of the collected data illuminated key aspects of the moral experience of the participants including clarifying and examining motivations and expectations, the relationality of humanitarian work, addressing steep imbalances of power, acknowledging and confronting the limited horizon of what can be accomplished and recognising the ways that organisational structures shape the everyday moral experience of practitioners. The focus on moral experience allowed access to a wide range of experiences by the participants in which values that they deemed important were realised or thwarted. Participants discussed concerns ranging from the effect of security protocols on patient care, to the impact of a history of colonialism on modern humanitarian aid initiatives, to the crucial role of project teams as key reference points and sources of psychological support. The study provided insight into important features of the moral dimension of this field of healthcare practice, and the moral and social world of medical relief work.

Moral experience provides a valuable framework for empirical research in bioethics. An important question that arises for enquiries into moral experience and, indeed, for much empirical research in bioethics is the so-called is/ought problem ${ }^{34} 35$ - that is, research that identifies how individuals act in particular circumstances, what they believe about a particular moral issue or how they experience an ethically significant situation does not necessarily provide an epistemological footing to critique or support social practices or beliefs. Researchers who conduct studies of moral experience must confront the issue of what to do regarding the normative implications of what is learnt. We acknowledge this challenge, but will not try to answer these questions here.

The inherently personal and subjective nature of moral experience leads to an inherent limitation for empirical enquiry that has examination of moral experience as its focus: what is accessible through an interpretive enquiry is always partial. The qualitative researcher who sets out to study the moral experience of individuals who share a common association to a phenomenon of interest (eg, all having the same diagnosis and facing similar treatment choices) should acknowledge that their ability as a researcher to access the moral experience of these persons will always be fragmentary and incomplete. Even a thoughtful nuanced enquiry into the moral experience of another will only allow the enquirer to access traces of that experience.

\section{CONCLUSION}

The conception of moral experience that we have developed in this paper is intended to provide an angle of vision in bioethics enquiry to examine a broader spectrum of how the moral is experienced in the everyday lives of individuals and groups. Our account of moral experience draws on anthropological sources and is subjective and hermeneutic: 'Moral experience encompasses a person's sense that values that he or she deems important are being realised or thwarted in everyday life. This includes a person's interpretations of a lived encounter, or a set of lived encounters, that fall on spectrums of right-wrong, goodbad or just-unjust'. Moral experience, in this conceptualisation, arises in many aspects of daily life and not only in moments of choice or in challenging or distressing circumstances.

Moral experience can be used to orientate empirical enquiry in bioethics. Empirical bioethics studies that explore the moral experience of individuals who share a common link to a phenomenon of interest will provide a distinctive perspective to examine how individuals and groups experience the moral aspects of their lives. Such a study would be conducted according to an accepted qualitative research methodology and orientated by a research question that expressly identifies accessing moral experience as the study's objective.

Moral experience also provides a framework for a broad bioethics research agenda that goes beyond the evaluation of ethical dilemmas and processes of moral justification and decision-making. This is consistent with views expressed by commentators within and beyond bioethics who have called for deeper and more sustained attention within bioethics to a set of concerns, experiences and issues that better captures what is ethically at stake for individuals and communities.

We propose moral experience as a framework for guiding theoretical analysis and empirical enquiry in bioethics. We believe that expressly focusing on moral experience will bring into view issues that are of concern to bioethics and bioethicists, but that receive less attention owing to a dominant focus on dilemmas, problems and distress.

Acknowledgements The authors are grateful to Lisa Schwartz and Hillel Braude for their helpful comments on this manuscript.

Funding MRH's research is supported by a Canadian Institutes of Health Research post-doctoral fellowship.

Competing interests None.

Provenance and peer review Not commissioned; externally peer reviewed.

\section{REFERENCES}

1. Pincoffs E. Quandary ethics. Mind 1971;80:552-71.

2. Hoffmaster B. The forms and limits of medical ethics. Soc Sci Med 1994;39:1155-64.

3. Sherwin SA. Relational approach to autonomy in health care. In: Sherwin S, ed. The Politics of Women's Health: Exploring Agency and Autonomy. Philadelphia: Temple University Press, 1998:19-47.

4. Meyers D. Self, Society, And Personal Choice. New York: Columbia University Press, 1989.

5. Braunack-Mayer A. What makes a good GP? An empirical perspective on virtue in general practice. J Med Ethics 2005;31:82-7.

6. Caplan $\mathbf{A L}$. The morality of the mundane: ethical issues arising in the daily lives of nursing home residents. In: Kane RA, Caplan AL, eds. Everyday Ethics: Resolving Dilemmas in Nursing Home Life. New York: Springer, 1990:37-50.

7. Kleinman A. Moral experience and ethical reflection: can ethnography reconcile them? A quandary for the new bioethics. Daedalus 1999;128:69-97.

8. Farmer P, Gastineau Campos N. Rethinking medical ethics: a view from below. Dev World Bioethics 2004;4:17-41. 
9. de Vries R, Turner L, Orfali K, et al. eds. The View from Here: Bioethics and the Social Sciences. Oxford: Blackwell Publishing, 2007.

10. Borry P, Schotsmans P, Dierickx K. The birth of the empirical turn in bioethics. Bioethics 2005:19:49-71.

11. Ives J. 'Encounters with experience': empirical bioethics and the future. Health Care Anal 2008:16:1-6.

12. Mandelbaum M. The Phenomenology of Moral Experience. Baltimore, MD: Johns Hopkins University Press, 1969.

13. Hsin Yang L, Kleinman A, Link BG, et al. Culture and stigma: adding mora experience to stigma theory. Soc Sci Med 2007;64:1524-35.

14. Kleinman A, Benson P. Culture, moral experience and medicine. Mt Sinai J Med 2006;73:834-9

15. Taylor C. Malaise of Modernity. Toronto: Anansi Press, 1991

16. Heidegger M. Being and Time. San Francisco, CA: Harper Collins Publishers, 1962 (translated by J Macquarrie, E Robinson).

17. Gadamer HG. Truth and Method. New York, NY: Crossroad Publishing Company, 1960/1975 (translated by J Weinsheimer, D Marshall).

18. Taylor C. Sources of the Self: The Making of the Modern Identity. Cambridge, MA: Harvard University Press, 1989.

19. Geertz C. The Interpretation of Cultures. New York, NY: Basic Books, 1973.

20. Taylor C. Human Agency and Language: Philosophical Papers 1. Cambridge: Cambridge University Press, 1985.

21. Taylor C. Modern Social Imaginaries. Durham, NC: Duke University Press, 2004

22. Taylor C. Philosophy and the Human Sciences: Philosophical Papers 2. Cambridge: Cambridge University Press, 1985.

23. Grondin J. Introduction to Philosophical Hermeneutics. New Haven, CT: Yale University Press, 1991/1994 (translated by J Weinsheimer)
24. Damasio AR. Descartes' Error: Emotion, Reason and the Human Brain. London: Macmillan, 1994.

25. Hacker PMS. The conceptual framework for the investigation of emotions. Int Rev Psychiatry 2004:16:199-208.

26. Nussbaum M. Emotions as judgments of value. Yale J Criticism 1992;5:201-12.

27. Guba EG, Lincoln YS. Competing paradigms in qualitative research. In: Denzin NK, Lincoln YS, eds. Handbook of Qualitative Research. Thousand Oaks, CA: Sage, 1994:105-17.

28. Benner $\mathbf{P}$. The tradition and skill of interpretive phenomenology in studying health illness and caring practices. In: Benner P, ed. Interpretive Phenomenology: Embodiment, Caring and Ethics in Health and IIIness. Thousand Oaks, CA: Sage, 1994:99-127.

29. Thorne S. Interpretive Description. Walnut Creek, CA: Left Coast Press, 2008.

30. Lincoln YS, Guba EG. Naturalistic Inquiry. Beverly Hills, CA: Sage, 1985.

31. Morse JM, Barrett M, Mayan M, et al. Verification strategies for establishing reliability and validity in qualitative research. Int J Qual Methods 2002;1:13-22. http://ejournals.library.ualberta.ca/index.php/IJQM/article/view/4603/3756 laccessed 31 Jan 2011).

32. Carnevale FA, Alexander E, Davis M, et al. Daily living with distress and enrichment: the moral experience of families with ventilator-assisted children at home. Pediatrics 2006;117:e48-60.

33. Hunt MR. Moral experience of Canadian health care professionals in humanitarian work. Prehosp Disaster Med 2009:24:518-24.

34. Haimes E, Williams R. Sociology, ethics, and the priority of the particular: learning from a case study of genetic deliberations. Br J Sociol 2007;58:457-76.

35. Ives J, Draper H. Appropriate methodologies for empirical bioethics: it's all relative. Bioethics 2009;23:249-58.

\section{Have confidence in your decision making.}
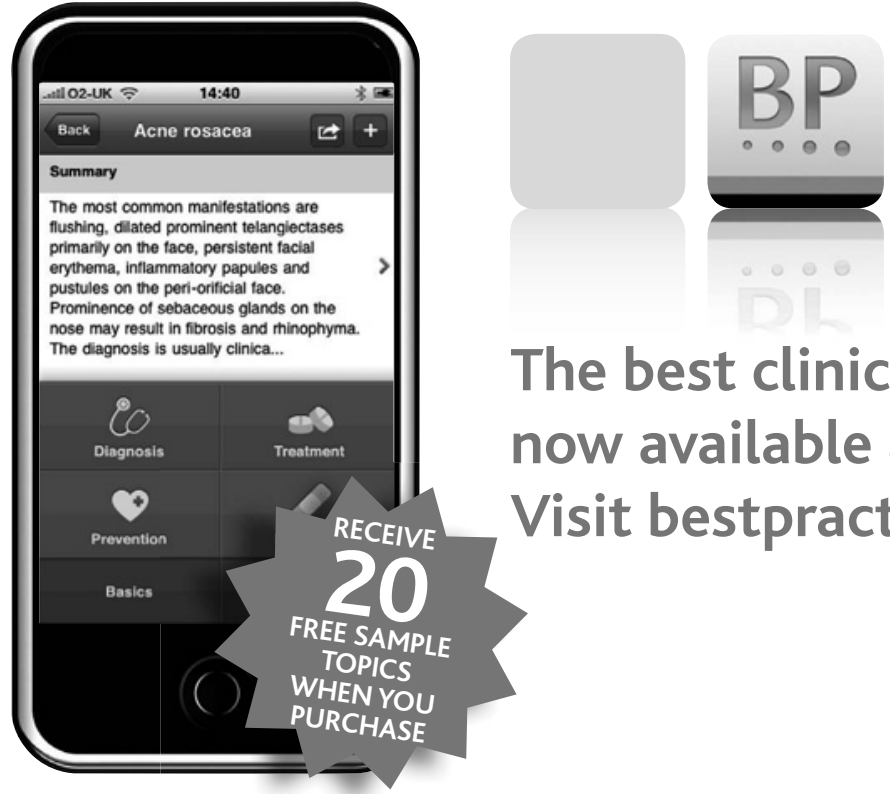

The best clinical decision support tool is now available as an app for your iPhone. Visit bestpractice.bmj.com/app

\section{clinicians $\bullet$ medical students $\bullet$ nurses $\bullet$ healthcare practitioners}

